\title{
A simulation analysis to optimize orbits for a tropical GPS radio occultation mission
}

\author{
Ashraf Mousa ${ }^{1}$, Yuichi Aoyama ${ }^{2 *}$, and Toshitaka Tsuda ${ }^{2}$ \\ ${ }^{1}$ National Research Institute of Astronomy and Geophysics, Helwan, Cairo 11422, Egypt \\ ${ }^{2}$ Research Institute for sustainable humanosphere, Kyoto University, Kyoto, Japan
}

(Received February 16, 2005; Revised December 19, 2005; Accepted June 7, 2006; Online published September 16, 2006)

\begin{abstract}
Space-based Radio Occultation (RO) measurements using a GPS receiver on a low Earth orbiter (LEO) provide accurate atmospheric refractivity profiles. EQUatorial Atmospheric Research Satellite (EQUARS) is a planned satellite mission carrying a GPS receiver for RO measurements, whose main focus is to study the vertical coupling process in the equatorial atmosphere and ionosphere through upward propagating atmospheric waves. This paper presents a model simulation to determine the best practical orbital parameters of a LEO satellite for GPS occultation, which provides dense occultation coverage from $20^{\circ} \mathrm{S}$ to $20^{\circ} \mathrm{N}$ and sparser coverage extending to $30^{\circ} \mathrm{S}$ and $30^{\circ} \mathrm{N}$. Constellations of 29 GPS satellites are computed every $10 \mathrm{sec}$ using the six Keplerian parameters based on real almanac data, while various orbits of LEO satellite are computed by varying orbital parameters, especially orbital altitude and inclination. Then, the occultation events are simulated under the assumption that the ray path between the occulting GPS and LEO satellites is a straight line. The simulation analysis shows that altitude and inclination angle of orbit are considered as principal parameters among the Keplerian parameters to accomplish the RO measurements in the equatorial region. Taking into account the long-lived mission, an avoidance of ionospheric F-layer influences, and practical antenna field of view, the best practical orbit for RO measurement in the equatorial region has an altitude of $750 \mathrm{~km}$ and an inclination of $20^{\circ}$. LEO on this orbit is expected to provide $530 \mathrm{RO}$ events per day. The analysis also shows that three LEOs in that orbit with $120^{\circ}$ separation can provide atmospheric profiles at least once every $6 \mathrm{~h}$ within $1000 \mathrm{~km}$ from an arbitrary station in the equator.
\end{abstract}

Key words: GPS, Low Earth Orbit (LEO) satellite, radio occultation, mission simulation.

\section{Introduction}

The space-based GPS RO technique is a promising tool for monitoring the Earth's atmosphere and ionosphere (e.g., Kursinski et al., 1997). The current paper presents the possibility of a GPS RO mission with a low Earth orbiter (LEO) whose focus is to have good coverage in and around the Earth's equator. The main focus is the area within the $\pm 20^{\circ}$ latitude, with a good coverage of the area within $\pm 30^{\circ}$ latitude. Several possible orbits of the LEO are simulated by varying the Keplerian parameters and the expected RO events as well as their longitudinal and latitudinal distributions based on the constellations of 29 GPS satellites are investigated. The LEO orbits are compared here to select the optimum orbit for the LEO mission in the equatorial region. Here, we mainly focus on the orbital altitude and its inclination, which are effective on the RO measurement. In this section, the basics of GPS RO technique are described and basic parameters of the EQUARS mission are introduced.

\footnotetext{
*Present address: National Institute of Polar Research, Itabashi, Tokyo, Japan.

Copyright (c) The Society of Geomagnetism and Earth, Planetary and Space Sciences (SGEPSS); The Seismological Society of Japan; The Volcanological Society of Japan; The Geodetic Society of Japan; The Japanese Society for Planetary Sciences; TERRAPUB
}

\subsection{Basics of GPS radio occultation}

When an electromagnetic signal passes through the atmosphere it is refracted. The magnitude of the refraction depends on the refractivity gradient normal to the signal path, which in turn depends on the density gradients and water vapor (in the lower atmosphere), as well as electron density (in the ionosphere). Thus, measurements of refraction will contain information on the density in the neutral atmosphere (and hence temperature and water vapor) and the electron density along the path (e.g., Kursinski et al., 1997). A series of such paths at different tangent heights, as shown in Fig. 1, yields measurements containing information on the vertical profile of refractivity. If the tangent heights lay within the neutral atmosphere, the refractivity can be converted to a profile of temperature and/or water vapor (e.g., Eyre, 1994). On the other hand, if tangent heights are above the neutral atmosphere, the refractivity profile is converted to a profile of electron density.

At radio frequencies, it is not possible to make direct geometric measurements of the refracted angle precisely. However, if the transmitter and receiver are in relative motion, the refraction introduces a change in the Doppler shift of the received signal, and this can be related to the refracted angle. Space-based RO measurements using GPS receivers on a LEO satellite provides accurate atmospheric refractivity profiles (e.g., Kursinski et al., 1997). 


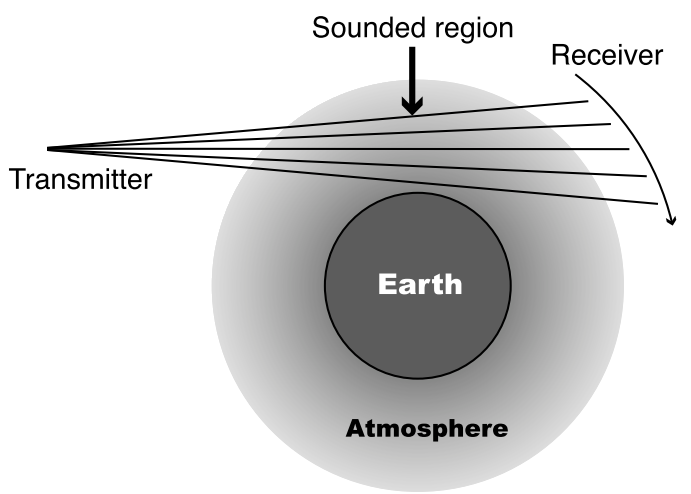

Fig. 1. Concept of refractivity profiling using the GPS radio occultation technique.

The GPS RO technique is an active limb sounding of the atmosphere and ionosphere. The occultation technique uses GPS navigation signals received on the LEO satellite. The unique characteristics of the RO technique include longterm stability, high accuracy, all-weather capability, global coverage, and high vertical resolution resolving a small atmospheric structure.

The GPS RO technique was demonstrated by the proofof-concept mission GPS/MET (Global Positioning System/Meteorology) experiment conducted by UCAR (University Corporation for Atmospheric Research) (Ware et al., 1996). GPS/MET has successfully provided the profiles of humidity and atmospheric temperature in the troposphere and stratosphere from April 1995 to February 1997 (Rocken et al., 1997). GPS/MET data are also used to study the electron density fluctuations in the ionosphere (e.g., Tsuda et al., 2004). The success of the GPS/MET experiment motivated the scientific community to launch many other LEO satellites to study the Earth's atmosphere and ionosphere.

The German CHAMP (CHAllenging Mini-satellite Payload) and the Argentinian SAC-C (Satellite de Aplicaciones Cientificas-C) satellites were launched on 15 July 2000 and 21 November 2000, respectively (Wickert et al., 2001). The data sets provided by these two missions are very useful for studying the detailed behavior of the atmosphere and ionosphere. In addition, the COSMIC (Constellation Observing System for Meteorology, Ionosphere and Climatology) with its six LEO satellites was launched in March 2006, while GRAS-SAF onboard METOP should be launched in April 2006 (Rocken et al., 2000; Larsen et al., 2004).

Most of the LEO satellites for RO launched so far have high orbital inclination angles. CHAMP orbits the Earth at $400 \mathrm{~km}$ altitude and $87^{\circ}$ inclination. The altitude of SAC$\mathrm{C}$ is $705 \mathrm{~km}$ while its inclination is $98.3^{\circ}$. The inclination of the COSMIC LEO satellites will be $72^{\circ}$ and their final altitudes will be $800 \mathrm{~km}$. Since inclination angles of these satellites are high, the distribution of the GPS occultation is concentrated in middle and high latitude regions and is relatively sparse in the low latitude region. With the objective of obtaining the dense observations in the equatorial region, a low inclination LEO is tested here. This study is needed as the LEO satellite with a focus on the equator with a low inclination angle such as EQUARS is now in the planning

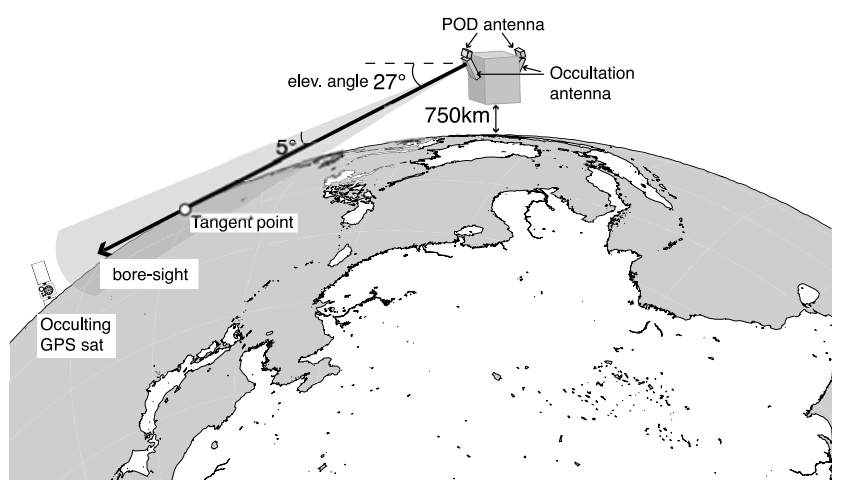

Fig. 2. Schematic diagram of EQUARS showing the attached GPS antennas. There are two sets of occultation and POD antennas, one set in the front and the other at the back.

stage.

\subsection{EQUARS radio occultation concept}

EQUARS is a mission planned for launching in 2007 by the Brazilian Space Agency (INPE) in collaboration with RISH (Research Institute of Sustainable Humanosphere), Kyoto University, Japan, Canada and USA (Tsuda et al., 2004). The main focus of EQUARS is to study the vertical coupling process in the equatorial atmosphere through upward propagating atmospheric waves.

The scientific mission of EQUARS as defined by the satellite project committee of INPE, is global-scale monitoring of the Earth's equatorial atmosphere and ionosphere. The main objective of the EQUARS is to study dynamical, photochemical, and ionospheric processes, with special emphasis on vertical energy transport by propagation of gravity, tidal, and planetary waves. EQUARS also aims to clarify the generation and development of plasma bubbles in the ionosphere. EQUARS will have eight scientific instruments to fulfill its mission, including an airglow imager for studying the gravity waves, ionospheric and plasma sensors for monitoring the ionosphere, as well as the GPS receiver.

For the RO experiment purposes, a GPS receiver will be installed on EQUARS, which will carry two positioning antennas as well as two occultation antennas (Fig. 2). The positioning antennas will be used for precise EQUARS orbit determination and Total Electron Contents (TEC) measurements. The occultation antennas will be attached to the front and back of EQUARS. This configuration is nearly the same as that employed for COSMIC, which will obtain both rising and setting occultations coming to the front and aft antennas.

This paper is organized into three sections. The method of orbital simulation is given in Section 2. Section 3 gives examples of the results and their meaning for the number and distribution of occultation events. Finally, a summary and conclusions of the research are presented in Section 4.

\section{Simulation Procedure}

In order to test the distribution and number of occultation events that a LEO is expected to provide, the GPS satellites' position needs to be calculated and the LEO satellite orbit needs to be assumed. Supposing that a satellite moves only under the action of a central force, its simplified posi- 


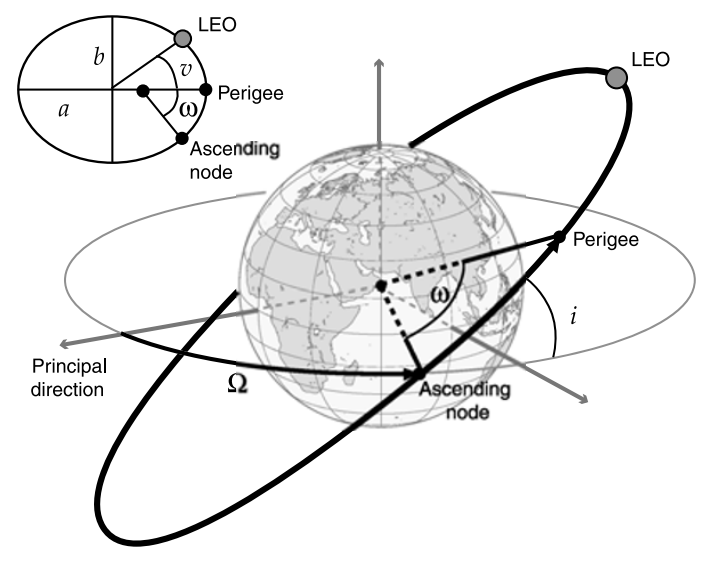

Fig. 3. Satellite orbital Keplerian parameters; the right ascension of the ascending node $(\Omega)$, inclination of the orbital plan $(i)$, the argument of perigee $(\omega)$, mean anomaly $(v)$, the semimajor axis $(a)$, and semiminor axis $(b)$. The upper-left part of the figure explains the semimajor and semiminor axes of the orbital ellipse (after Xu, 2003).

tion is defined by Keplerian motion with the six Keplerian parameters (Fig. 3). For GPS, the almanac data (predicted Keplerian parameters) provided by the navigation data center of the USA Coast Guard is used as the starting point for calculating the GPS satellites position.

Starting with the Keplerian orbital parameters, the GPS satellites' position is calculated in an Earth-Centered EarthFixed frame (ECEF) (for details of these calculations, refer to any GPS text book (e.g., Xu, 2003)). The LEO position is also calculated in the same way as the GPS satellites', but starting with assumed orbital parameters.

Several possible LEO orbital parameters are considered here to choose the best possible one. Here, the altitude will be considered as a positioning parameter for LEO rather than the semi-major axes (altitude $=$ semi-major axis-Earth's radius). The LEO altitude is varied among $3000,2500,2000,1500,1000,750$ and $500 \mathrm{~km}$. For each altitude, the seven different orbital inclination angles assumed here are 30, 25, 20, 15, 10, 5 and 0 degrees. Since LEO orbit is assumed as a circular orbit, the eccentricity is set to zero. In addition, the right ascension of the orbit-ascending node, the argument of perigee and the mean anomaly of the LEO are assumed to be zero and they do not vary (except for the mean anomaly in Section 3.4).

For reference and comparison against high inclination, the 72 and $90^{\circ}$ inclination are considered. This is also helpful in assessing the contribution of both COSMIC and CHAMP mission for studying the Earth's equatorial region. As stated before, the actual inclination of CHAMP is $87^{\circ}$, but here the $90^{\circ}$ inclination is considered for the sake of being general. Also, the difference between 87 and $90^{\circ}$ inclination is small enough and will not change the results of the simulation.

GPS orbital period is $11 \mathrm{~h}$ and $58 \mathrm{~min}$, and, as a result, the satellites appear at the same position everyday about 4 min earlier. It takes one whole year for the GPS satellites to appear again in the same position and exact time. To make a complete simulation means to repeat the analysis for the whole year. This is not practical and is troublesome when computer CPU time is considered. To make the data sta- tistically significant, the simulation was carried out for the first 8 days every two months. January, March, May, July, September, and November of the year 2004 were chosen for the analysis. Thus, the analyses are considered for 48 days, i.e. about $13 \%$ of the whole year.

The actual ray propagating from GPS to LEO is bent, due to the atmosphere. For simulation, the signal propagation from GPS to LEO is approximated here by a straight line. This approximation seems reasonable as the maximum expected bending due to the Earth's atmosphere is about $1^{\circ}$, which is considered to be small and unimportant for this analysis. Also the straight line assumption is suitable for making fast and simple calculations considering the alternative in the implementation of the complicated and expensive ray tracing algorithms.

All the possible rays connecting the GPS to the LEO are considered here. The ray is considered to be an occultation event if its elevation angle is in the range of $\pm 5^{\circ}$ of the occultation antenna bore sight direction. Also, the limits on the tangent point height are taken between 80 and $150 \mathrm{~km}$ for defining a single occultation event. The threedimensional position of the start and end points of an occultation event are recorded for the analysis. The sampling rate is set to $10 \mathrm{sec}$. Unless otherwise mentioned, both rising and setting occultation arriving at the front and aft antennas will be considered in the analysis.

\section{Results and Discussion}

The number of occultation events and their distribution are considered here as the criteria for comparing different LEO possible orbits. The results of Sections 3.1, 3.2, and 3.4 show the case restricting the antenna field of view (FOV) angle to $45^{\circ}$ on both sides of the orbit which is due to the assumed antenna gain pattern (this will be considered in more detail in Section 3.3). As mentioned before, we approximated the signal propagation by a straight line to ease the calculation. This approximation is expected to reduce the signal delay by more than $1 \mathrm{~km}$. and the tangent height will be lowered by about $50 \mathrm{~km}$. As only the number of occultation is considered here, this approximation is not expected to change the results.

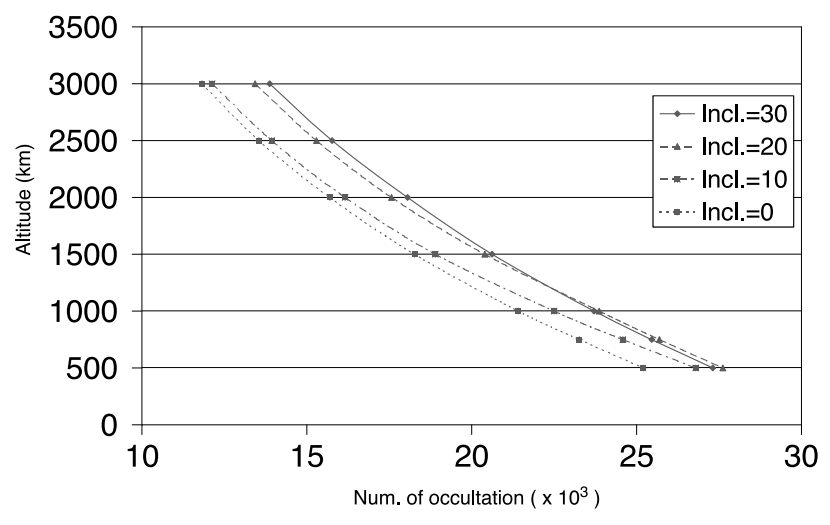

Fig. 4. Effect of changing the orbit altitude on the number of occultation events. The figure shows the total expected number of occultation events with an antenna FOV angle of about $45^{\circ}$ (this is equivalent to considering only gains greater than $-3 \mathrm{~dB}$ ) over the selected days. 


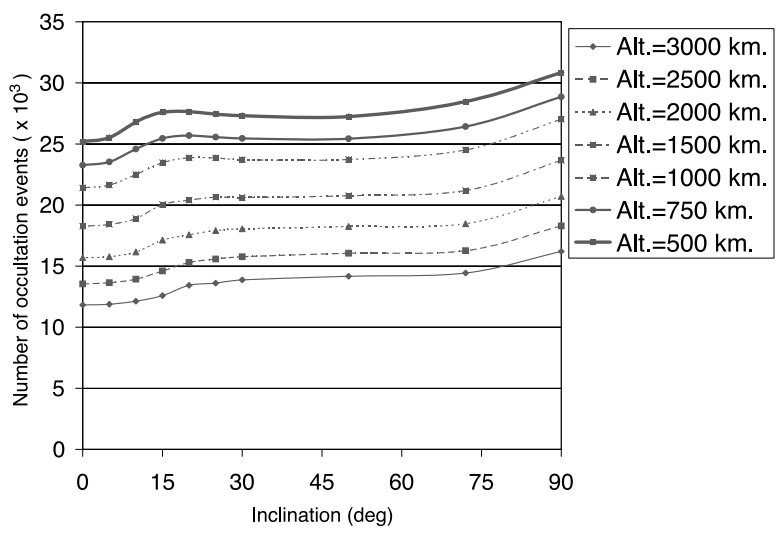

Fig. 5. Effect of changing the EQUARS orbital inclination on the number of occultation events. The figure shows the total expected number of occultation events whose gains are greater than $-3 \mathrm{~dB}$, over the selected days.

\subsection{Effect of orbit altitude}

Figure 4 shows the results for different orbit altitudes. In this figure, seven altitudes are considered at 3000, 2500, $2000,1500,1000,750$, and $500 \mathrm{~km}$. It is clear from Fig. 4 that, regardless of the orbital inclination, lowering the satellite altitude produces more occultation events. This curve also shows that increasing the inclinations leads to an increase in the number of occultation events. The 20 and $30^{\circ}$ inclination curves show more events for the $30^{\circ}$ inclination down to an altitude of about $1250 \mathrm{~km}$. However, for the lower altitude, an inclination of $20^{\circ}$ gives more events than that of $30^{\circ}$.

In reality, it is important to avoid any altitude lower than $300 \mathrm{~km}$ to insure the stability of the orbit and to avoid the ionospheric disturbance in the F-layer. At low latitude (around the geomagnetic equator), which is the case considered here, the F-layer effects occur at an altitude of about $400-500 \mathrm{~km}$. In addition, a lifetime of satellite mission depends on the atmospheric drag force, because it is related to the decay of orbital altitude. Since the atmospheric drag force is approximated by a linear function of an atmospheric density and a satellite shape, the atmospheric drag force at an altitude of $750 \mathrm{~km}$ is expected to be smaller than that at an altitude of $500 \mathrm{~km}$ by an order of magnitude in the case of the same satellite (shape). Thus, the altitude of $750 \mathrm{~km}$ is considered to be the practical one for the LEO considered here. This will also allow more accurate observations of the ionosphere, as the signal will travel a longer path through the ionosphere.

\subsection{Effect of orbit inclination}

Figure 5 shows the effect of the inclination on the total number of events at different altitudes. It is clear that all the curves follow the similar trend of increasing gradually with increasing of the inclination until it gives a global maximum at $90^{\circ}$ inclination. Figure 5 also indicates that for altitudes above $1500 \mathrm{~km}$, the number of occultation events increase monotonically with increasing the LEO inclination angle till its maximum at $90^{\circ}$. On the other hand, there is a local maximum in the number of occultation events by varying the inclination angle at fixed altitudes lower than $1500 \mathrm{~km}$. For example, the altitude of $750 \mathrm{~km}$ has its local maximum
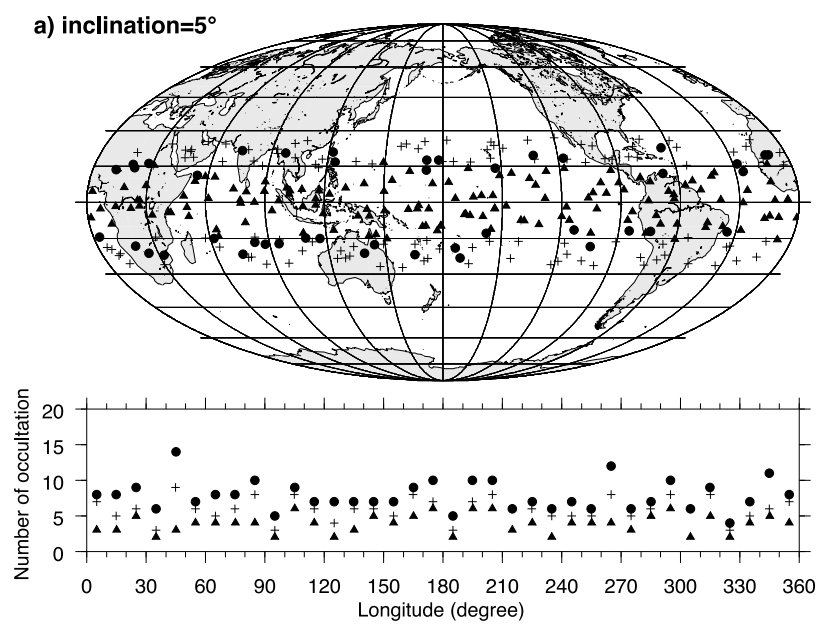

b) inclination $=20^{\circ}$
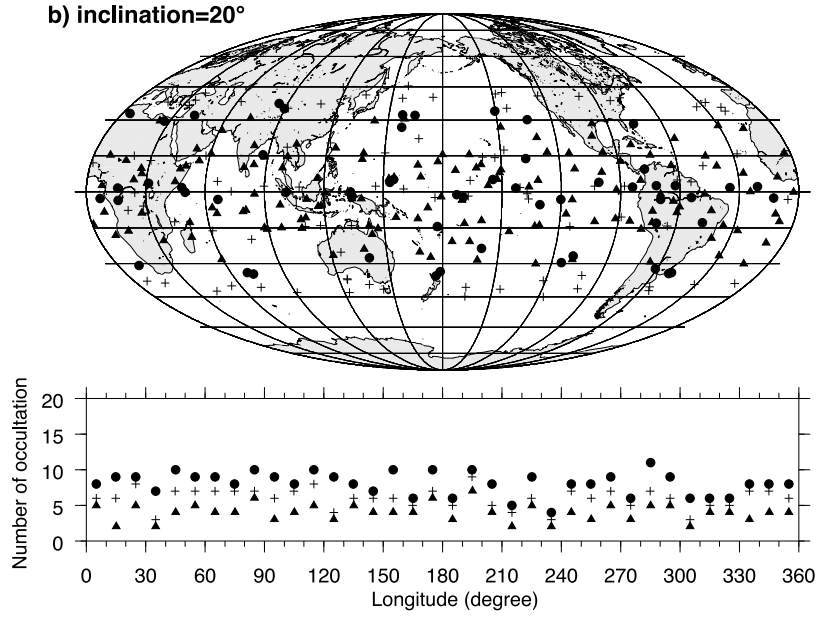

Fig. 6. Example of longitudinal distribution of expected occultation events for a LEO with a $750 \mathrm{~km}$ altitude and one occultation antenna. The figure shows the distribution of the expected occultation events for 1 January 2004 in two orbit inclinations. Part (a) shows the results for the $20^{\circ}$ inclination while part (b) shows the results for the $5^{\circ}$ inclination. The solid circle shows the expected events within the $-3 \mathrm{~dB}$ antenna gain, the cross curve is the events within $-2 \mathrm{~dB}$ antenna gain and the solid triangle one is for $-1 \mathrm{~dB}$ antenna gain.

at about $20^{\circ}$ inclination angle.

In order to have a clear idea about which inclination is suitable for the LEO considered here, we investigate the longitudinal and latitudinal distribution of events. Figure 6 shows an example of the occultation event distributions on a global map as well as their longitudinal distributions acquired with the front antenna, for two orbital inclinations 20 and $5^{\circ}$ for 1 January 2004 . This figure shows that the longitudinal distribution of the events is nearly homogeneous, regardless of the orbital inclination angle. Considering the solid circle curve (antenna FOV angle of $45 \mathrm{deg}$ ), both inclinations provide about 10 events daily every $10^{\circ}$ in longitude. Comparing the distribution of events on the global map for the two inclinations indicates that the $20^{\circ}$ inclination shows better latitude coverage up to $\pm 30^{\circ}$, while the events provided with $5^{\circ}$ inclination is much more concentrated around the $\pm 10^{\circ}$ latitude.

Figure 7 shows latitudinal distributions of the expected occultation events for the whole (48 days in total) data set 


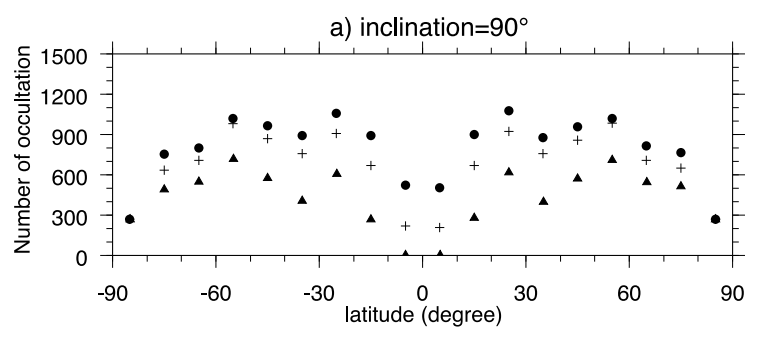

b) inclination $=72^{\circ}$

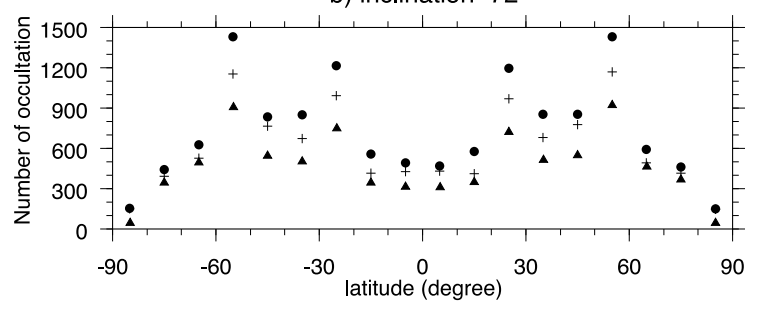

c) inclination $=20^{\circ}$

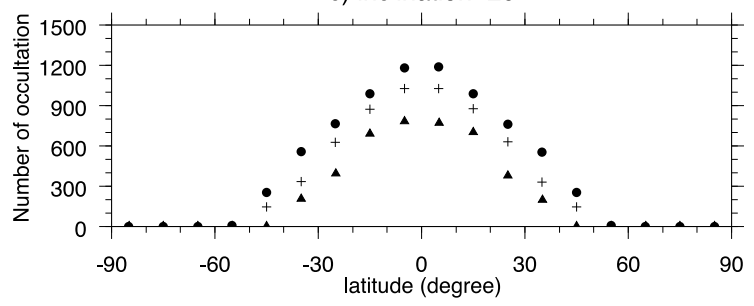

d) inclination $=5^{\circ}$

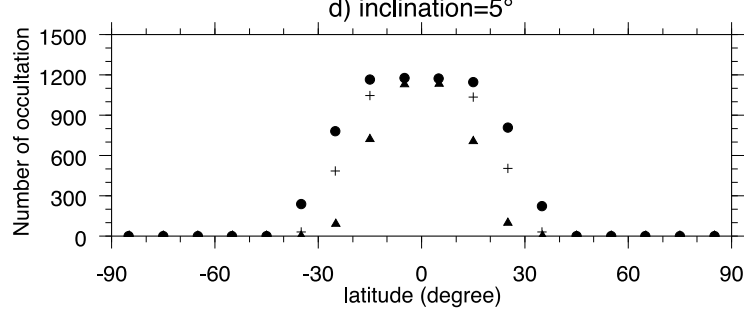

Fig. 7. The latitudinal distribution of expected occultation events for a LEO with a $750 \mathrm{~km}$ altitude and one occultation antenna. The distribution for four orbit inclinations; using whole (48 days in total) data set is shown here. Parts (a), (b), (c), and (d) show the results for 90, 70, 20 , and $5^{\circ}$ inclination angles, respectively. The solid circle shows the expected events within the $-3 \mathrm{~dB}$ antenna gain, the cross curve is the events within the $-2 \mathrm{~dB}$ antenna gain and the solid triangle one is for the $-1 \mathrm{~dB}$ antenna gain.

considered with a $10^{\circ}$ latitudinal bin for four possible orbital inclinations, 90, 72, 20 and $5^{\circ}$. Here only the solid circles curve will be considered, which shows the case for considering the $45^{\circ}$ restricted antenna FOV. All four inclinations give a distribution that decreases and reach a minimum value of about 300 or less at latitudes poleward of $80^{\circ}$.

An inclination of $90^{\circ}$ gives the latitudinal distribution that has its maximum value of about 1000 events (about 21 events/day/antenna) at latitudes north or south of $30^{\circ}$ and shows a local minimum of about 900 events (about 19 events/day/antenna) around the equator. The occultation event distribution for the $72^{\circ}$ inclination angle shows a maximum value of 1400 events (about 29 events/day/antenna) at latitudes north and south of $55^{\circ}$ and a minimum value of

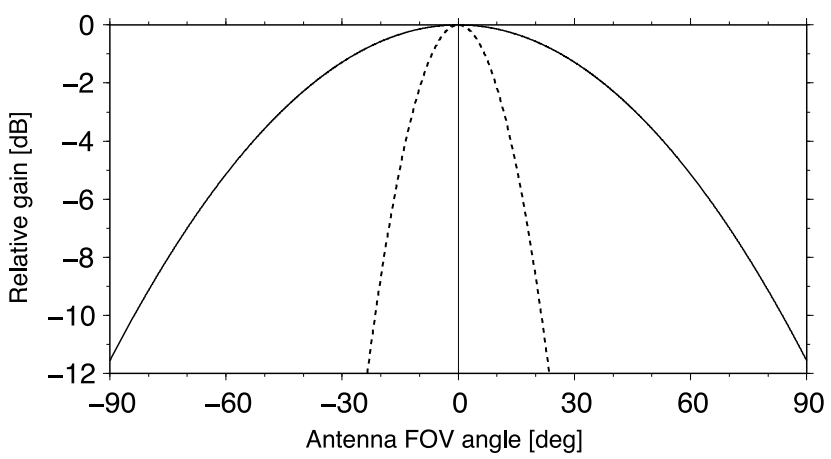

Fig. 8. The assumed antenna gain patterns. The curves show the gain relative to $13 \mathrm{~dB}$ at the bore-sight direction as a function of the antenna FOV angle for elevation (dotted line) and azimuth (solid line) directions.

about 600 events (about 12 events/day/antenna) at the equator and up to a latitude of about $\pm 20^{\circ}$.

On the other hand, for an inclination of $5^{\circ}$, the distribution is concentrated around the equator with a maximum number of about 1100 events (about 23 events/day/antenna). For the $20^{\circ}$ inclination case, the maximum number is about 1200 events (about 25 events/day/antenna) at the equator up to $\pm 10^{\circ}$ latitude. Comparing the distribution for the inclination of 5 and $20^{\circ}$, one can see that the $20^{\circ}$ inclination angle distribution gives a maximum density more than that possible with the $5^{\circ}$ inclination in the tropics $\left(30^{\circ} \mathrm{S}\right.$ to $\left.30^{\circ} \mathrm{N}\right)$.

Realizing that the aim of the LEO considered here is to cover the tropics, it is obviously not suitable to choose a high inclination. Also, for having maximum coverage for this region, it is better to choose the $20^{\circ}$ inclination. This is compatible with the results indicated by Fig. 5 that the local maximum point for the $750 \mathrm{~km}$ altitude is at the $20^{\circ}$ inclination.

As stated above, only the solid circle curve in Figs. 6 and 7 is considered in the above discussion. The solid circle curve represents the number of possible events if we consider only events within the $45^{\circ}$ antenna FOV angle. The solid triangle and cross curves are considered in the next section dealing with the antenna gain and FOV. However, it is interesting to note that the triangle and the circle curves follow nearly the same trend as the continuous line curve and thus support the same results about the orbital inclination.

It is important to note here that in both Figs. 6 and 7, only the distribution of the events for one antenna is considered. The results show that the other antenna provides almost the same distribution with minor differences due to the geometry of the GPS satellite. These differences are statistically insignificant. Considering only one antenna is also helpful in assessing the CHAMP satellite contribution to areas around the equator as it has only one antenna.

\subsection{Effect of restricting the antenna field-of-view angle (antenna gain)}

The bore sight angle is strongly correlated with the antenna gain. The antenna gain pattern (normalized relative to the bore-sight gain) of the assumed antenna is shown in Fig. 8 as a function of the antenna FOV angle. Figure 8 also shows that, for the antenna considered here, the $-3 \mathrm{~dB}$ an- 


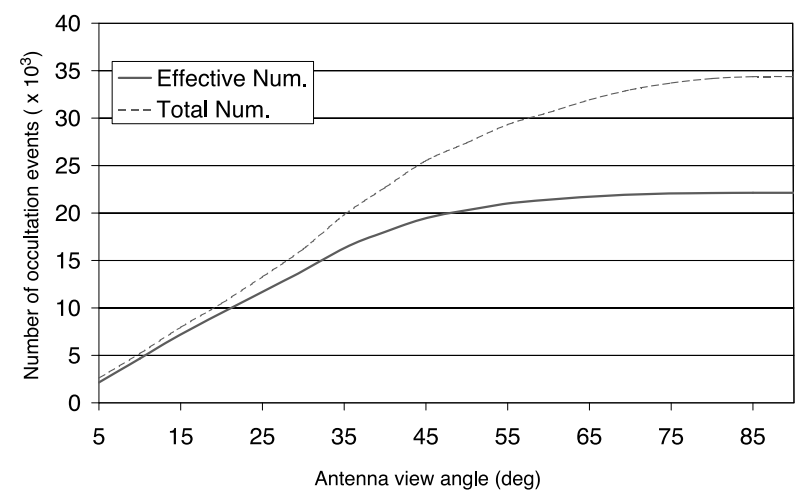

Fig. 9. Effect of antenna gain on the number of occultation events.

tenna gain is equivalent to restricting the assumed antenna FOV angle to $45^{\circ}$ both ways from bore-sight. The $-2 \mathrm{~dB}$ antenna gain is equal to $37^{\circ}$ restricted antenna FOV. The $-1 \mathrm{~dB}$ antenna gain limit is the case for $26^{\circ}$ restricted antenna FOV.

The simulation results suggest that for the $-1 \mathrm{~dB}$ antenna gain (solid triangle in Figs. 6 and 7), about 56\% on average is lost out of the total expected number of events. The loss is about 40 and $25 \%$ for the $-2(+$ curve) and $-3 \mathrm{~dB}$ (solid circle), respectively.

The effect of antenna gain pattern is considered in Fig. 9 in terms of the antenna FOV angle. The dashed curve shows the accumulated number of the expected events over the whole data sets without any effect of the antenna gain. The dashed curve indicates that the number of events increase with a widening of the antenna FOV angle up to about $65^{\circ}$. After $65^{\circ}$, the curve is almost flat, which indicates saturation and widening the antenna FOV angle more does not increase the number of events.

If a linear relation is assumed between the number of observed events and the antenna gain, the number of events will follow the solid curve in Fig. 9. The solid curve indicates that at $45^{\circ}$ antenna FOV angle we lose about $23 \%$ of the total number of occultation events. Also, the saturation happens much earlier at the antenna FOV angle of $45^{\circ}$.

It is important to note here that with decreasing antenna gain, the signal-to-noise ratio (SNR) of the signal also decreases. The GPS receiver might be able to track down into the atmosphere until a certain critical SNR is reached. Since the number of occultation events shows saturation at the $45^{\circ}$ FOV angle of antenna, which means $-3 \mathrm{~dB}$, it might be possible as a first approximation to consider any lower gain out of the range of detection of the GPS receiver. This will not decrease the effective number of occultation events which can be deduced from the continuous curve of Fig. 9 .

\subsection{Effect of using more than one satellite in the same} orbit

In order to test the number of expected occultation events over a certain (local) area, the above-chosen best practical orbit is used. One LEO is considered and the number of events falling within $500 \mathrm{~km}$ of distance from a chosen point (a specific ground-based observatory) is counted. On average, every $12 \mathrm{~h}$, one occultation event is possible. In an effort to increase the number of events within a local

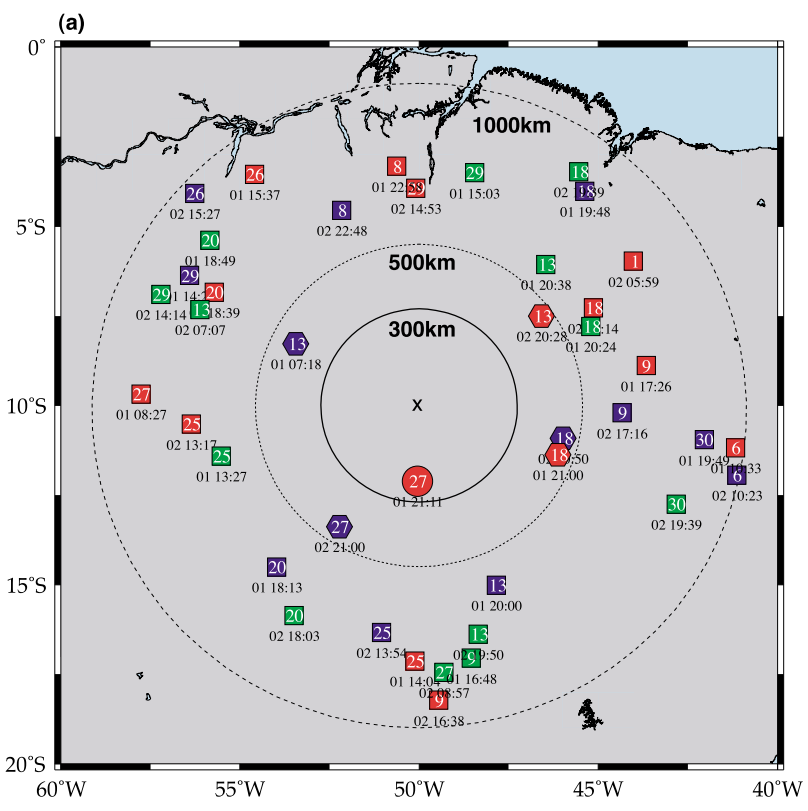

(b) Time table of occultation events

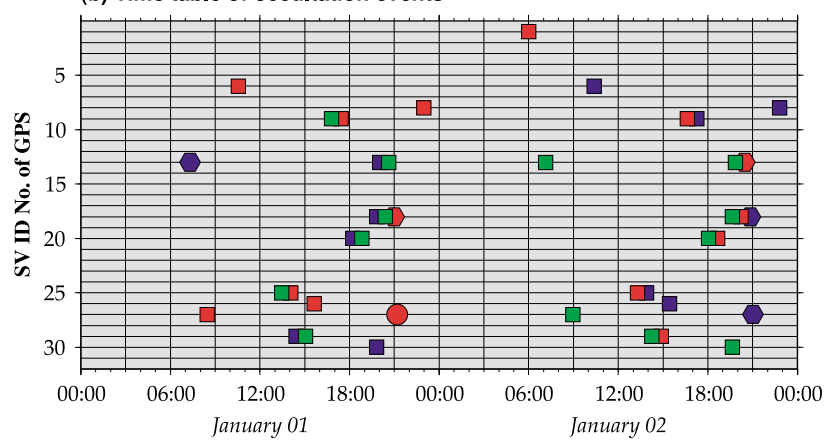

\begin{tabular}{l}
\begin{tabular}{|l|l|l|l|}
\hline \multicolumn{4}{|c|}{ 41 events during 2004-01-01 00:00 - 2004-01-02 23:59 } \\
\hline Sat: & $\bullet$ Leo 1 & $\bullet$ Leo 2 & $\bullet$ Leo 3 \\
\hline D from $\left(50^{\circ} \mathrm{W}, 10^{\circ} \mathrm{S}\right)$ & $\bigcirc 0-300 \mathrm{~km}$ & $300-500 \mathrm{~km}$ & $\square 500-1000 \mathrm{~km}$ \\
\hline
\end{tabular} \\
\begin{tabular}{|l} 
The number within above marks indicates SV ID No. of GPS satellites. The time below the marks \\
is a middle time of each occultation events.
\end{tabular} \\
\hline
\end{tabular}

Fig. 10. An example of the effect of having more satellites in the same orbit on the total number of occultation events. Three equally spaced satellites coverage is shown around the station located at $\left(10^{\circ} \mathrm{S}, 50^{\circ} \mathrm{W}\right)$ in Brazil. Part (a) shows the distribution of events within 300, 500 and $1000 \mathrm{~km}$ distances from the station for 1st and 2nd of January 2004. Part (b) shows the time distribution while the lower part explains the symbol used in the figure.

area, the effect of having more than one satellite in the same orbit is also checked with two configurations. The first is equally spaced satellites, while the second configuration is very close satellites in the same orbit. Two, three, four, five, and six satellites were considered. For the very close satellite, they were defined to make flights separated by $36^{\circ}$ in mean anomaly, which corresponds to an almost $10 \mathrm{~min}$ time difference at a $750 \mathrm{~km}$ orbit altitude. The analysis was stopped at six satellites as more than that are not considered to be visible.

An example of an observatory located at $10^{\circ} \mathrm{S}$, and $50^{\circ} \mathrm{W}$ in Brazil is given in Fig. 10. This figure shows the occultation events obtained from three equally spaced satellites separated by $120^{\circ}$ in the same orbital plane for two days; 1 and 2 January 2004. In this case, at least one atmospheric profile will be obtained every $6 \mathrm{~h}$ within $1000 \mathrm{~km}$ from the observatory. This simulation result suggests that the GPS 
occultation measurements with the multiple LEOs have the ability to measure atmospheric profiles comparable to temporal and spatial resolution of the radiosonde observation.

Analysis of the whole 48-day data sets reveals that the increase in the number of events is linearly proportional to the number of satellites, regardless of whether they are near or equally spaced. The difference between nearly spaced and equally spaced satellites is that with nearly spaced satellites the events are more concentrated, both spatially and temporarily.

\section{Conclusions}

Simulation of different possible orbits for a GPS radio occultation LEO mission with a concentration on tropical coverage is shown here. The GPS satellite position is calculated using broadcast almanac data. The LEO altitude, inclination, its antenna view angle and gain are considered here. Number of occultation events and their longitudinal and latitudinal distributions are the parameters for comparing different possible LEO orbits. The possibility of increasing the occultation numbers over a given area is also studied.

The analysis showed that the most practical orbital plan for LEO is the one with $750 \mathrm{~km}$ altitude and $20^{\circ}$ inclination. An antenna view angle wider than $45^{\circ}$ will have lower SNR and thus might fall out of the range of the receiver detection. The antenna gain effect on the number of events as well as the receiver detection limit need to be investigated further using experiences gained from other missions like CHAMP or SAC-C satellites. It is also shown that the number of expected events increase linearly with increasing number of satellites in the same orbit.

Acknowledgments. This study was promoted as a part of the project of "Application of Precise Satellite Positioning for Monitoring the Earth's Environment" supported by the Ministry of Ed- ucation, Culture, Sports, Science and Technology, Japan (MEXT). The first author wishes to express his thanks to the Japan Student Services Organization, JASSO, for offering a chance to stay and carry out this research at the Research Center for Sustainable Humanosphere, Kyoto University as a visiting scientist.

\section{References}

Eyre, J. R., Assimilation of radio occultation measurements into a numerical weather prediction system, Tech. Memo 199, 22 pp., Eur. Cent. for Medium-Range Weather Forecasts, Reading, England, 1994.

Kursinski, E. R., G. A. Hajj, K. R. Hardy, J. T. Schofield, and R. Linfield, Observing Earth's atmosphere with radio occultation measurement using the Global Positioning System, J. Geophys. Res., 102, 2342923465, 1997.

Larsen, G. B., K. B. Lauristen, F. Rubek, and M. B. Sorensen, GRAS-SAF Radio Occultation Data from EPS/Metop, in Occultations for Probing Atmosphere and Climate, edited by G. Kirchengast, U. Foelsche, and A. K. Steiner, pp. 111-118, Springer, Germany, 2004.

Rocken, C., R. Anthes, M. Exner, D. Hunt, S. Sokolovskiy, R. Ware, M. Gorbunov, W. Schreiner, D. Feng, B. Herman, Y.-H. Kuo, and X. Zou, Analysis and validation of GPS/MET data in the neutral atmosphere, $J$. Geophys. Res., 102, 29849-29866, 1997.

Rocken, C., Y.-H. Kuo, W. Schreiner, D. Hunt, S. Sokolovskiy, and C. McCormick, COSMIC System Description, Terrestrial Atmos. Oceanic Sci., 11, 21-52, 2000.

Tsuda, T., K. Hocke, and H. Takahashi, Utility of occultations for atmospheric wave activity studies: results of GPS/MET data analysis and future plan, in Occultations for Probing Atmosphere and Climate, edited by G. Kirchengast, U. Foelsche and A. K. Steiner, 345-352, Springer, Germany, 2004.

Ware, R., M. Exner, D. Feng, M. Gorbunov, K. Hardy, B. Herman, Y. Kuo, T. Meehan, W. Melbourne, C. Rocken, W. Schreiner, S. Sokolovskiy, F. Solheim, X. Zou, R. Anthes, S. Businger, and K. Trenberth, GPS sounding of the atmosphere from Low Earth Orbit: preliminary results, Bull. Am. Meteorol. Soc., 77, 19-40, 1996.

Wickert, J., C. Reigber, G. Beyerle, R. Konig, C. Marquardt, T. Schmidt, L. Grunwaldt, R. Galas, T. K. Meehan, W. G. Melbourne, and K. Hocke, Atmospheric sounding by GPS radio occultations: first results from CHAMP, Geophys. Res. Lett., 28, 3263-3266, 2001.

$\mathrm{Xu}, \mathrm{G} .$, GPS Theory, Algorithms and Applications, 315 pp., Springer, Germany, 2003.

A. Mousa (e-mail: ashrafkm@yahoo.com), Y. Aoyama, and T. Tsuda 